\title{
Loose-fit polypseudorotaxanes constructed from Y-CDs and PHEMA-PPG-PEG-PPG-PHEMA
}

\author{
Tao Kong, Lin Ye, Ai-ying Zhang and Zeng-guo Feng*
}

\author{
Full Research Paper \\ Address: \\ School of Materials Science and Engineering, Beijing Institute of \\ Technology, Beijing 100081, China \\ Email: \\ Zeng-guo Feng ${ }^{*}$ - sainfeng@bit.edu.cn \\ * Corresponding author \\ Keywords: \\ block copolymer; $\mathrm{Y}$-CD; loose-fit; poly(2-hydroxyethyl methacrylate); \\ polypseudorotaxane
}

Beilstein J. Org. Chem. 2014, 10, 2461-2469.

doi:10.3762/bjoc. 10.257

Received: 11 July 2014

Accepted: 24 September 2014

Published: 23 October 2014

This article is part of the Thematic Series "Superstructures with cyclodextrins: Chemistry and applications II".

Guest Editor: G. Wenz

(C) 2014 Kong et al; licensee Beilstein-Institut. License and terms: see end of document.

\begin{abstract}
A pentablock copolymer was prepared via the atom transfer radical polymerization of 2-hydroxyethyl methacrylate (HEMA) initiated by 2-bromoisobutyryl end-capped PPO-PEO-PPO as a macroinitiator in DMF. Attaching PHEMA blocks altered the selfassembly process of the pentablock copolymer with $\gamma$-CDs in aqueous solution. Before attaching the PHEMA, the macroinitiator was preferentially bent to pass through the inner cavity of $\gamma$-CDs to give rise to tight-fit double-chain stranded polypseudorotaxanes (PPRs). After attaching the PHEMA, the resulting pentablock copolymer was single-chain stranded into the interior of $\gamma$-CDs to form more stable, loose-fit PPRs. The results of ${ }^{1} \mathrm{H}$ NMR, WXRD, DSC, TGA, ${ }^{13} \mathrm{C}$ CP/MAS NMR and FTIR analyses indicated that $\gamma$-CDs can accommodate and slip over PHEMA blocks to randomly distribute along the entire pentablock copolymer chain. This results in unique, single-chain stranded PPRs showing no characteristic channel-type crystal structure.
\end{abstract}

\section{Introduction}

Cyclodextrins (CDs) are a family of cyclic oligosaccharides composed of 6,7 or 8 glucose units linked via $\alpha$-1,4-glycosidic bonds. Due to the presence of a hydrophobic inner cavity with different geometric dimensions, CDs can act as host molecules to not only small guest molecules, but also to linear polymeric guest molecules. They can self-assemble into novel inclusion complexes (ICs), or polypseudorotaxanes (PPRs) and polyrotaxanes (PRs) end-capped by bulky stoppers. For example, $\alpha$-CDs typically include PEG, but not PPG, $\beta$-CDs contain PPG instead of PEG, and $\gamma$-CDs accommodate either single-chain PPG or double-chain PEG [1]. The driving force behind the self-assembly is mostly ascribed to a suitable fit between the cross-sectional area of the incoming polymer chain and the cavity size of the CDs [2]. However, the cavity shape and size of CDs are deformable and variable to some extent. Their stability can vary depending on the solution, incoming guest molecules, and especially the number of glucose units (i.e., more glucose units give way to more structure flexibility) [3]. 
This possibly explains how Harada et al. [4] reported the first PPRs (comprised of $\gamma$-CD and PEG) as early as the 1990s. The $\gamma$-CD-based PPRs with designated supramolecular structure have been seldom prepared as compared with the $\alpha-C D$ - or $\beta$-CD-based PPRs [5-13].

Besides the typical double-chain stranded PPR showing a characteristic channel-type crystal structure, as reported by Harada et al. [4], the so-called single-chain stranded $\gamma$-CD-based PPRs or PRs have recently attracted tremendous attention. Their potential smart material and biomedical applications stem from their unique loose-fit rather than tight-fit structure of $\gamma$-CDs with a guest polymer [5]. Due to their superior deformability and adaptability, $\gamma$-CDs are able to slip over the bulkier PNIPAAm homo- and co-polymers to give rise to single-chain stranded, loose-fit PPRs or PRs showing no characteristic channel-type crystal structure [14-16]. To the best of our knowledge, self-assembled PPRs from $\gamma$-CDs with the bulkier poly(2hydroxyethyl methacrylate) (PHEMA)-flanked block copolymers have not yet been reported. Herein, a pentablock copolymer PHEMA-PPO-PEO-PPO-PHEMA is prepared via atom transfer radical polymerization (ATRP) in DMF, and allowed to self-assemble with $\gamma$-CDs in aqueous solution to form PPRs. The results of ${ }^{1} \mathrm{H}$ NMR, WXRD, DSC, TGA, ${ }^{13} \mathrm{C}$ $\mathrm{CP} / \mathrm{MAS}$ NMR and FTIR analyses indicate that the attachment of PHEMA clearly changes the self-assembly direction of $\gamma$-CDs with PHEMA-PPO-PEO-PPO-PHEMA. This results in unique, single-chain stranded, loose-fit PPRs, instead of the PEG-bent, double-chain stranded, tight-fit ones as shown in Scheme 1 .

\section{Results and Discussion}

\section{Self-assembly of $\mathrm{Y}-\mathrm{CDs}$ with a macroinitiator} and a pentablock copolymer in aqueous solution

As previously reported, a distal 2-bromoisobutyryl end-capped PEG was preferably bent to fit into the cavity of $\gamma$-CDs to form stable and unconventionally conformational PPRs. $\gamma$-CDs can accommodate the bulkier poly( $N$-isopropylacrylamide) (PNIPAAm) blocks to give single-chain stranded, loose-fit PPRs or PRs [14-16]. To extend the scope of self-assembly of $\gamma$-CDs with polymers having bulkier cross-sectional areas, PHEMA is attached to both ends of PPO-PEO-PPO by ATRP to yield a pentablock PHEMA-PPO-PEO-PPO-PHEMA copolymer. It is then used to investigate the possibility of selfassembly with $\gamma$-CDs $[17,18]$. The synthetic pathway for the pentablock copolymer is shown in Scheme 2. To shed light on the impact of end-capping groups on the self-assembly direction of $\gamma$-CDs with an incoming polymer chain, a PPO-PEO-

(1)

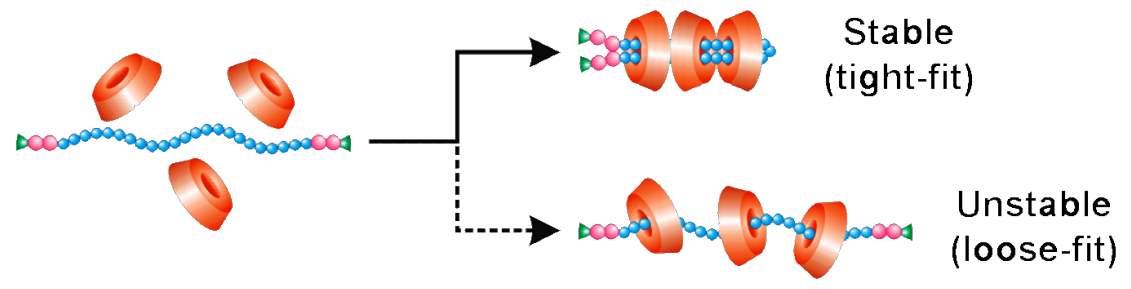

(2)
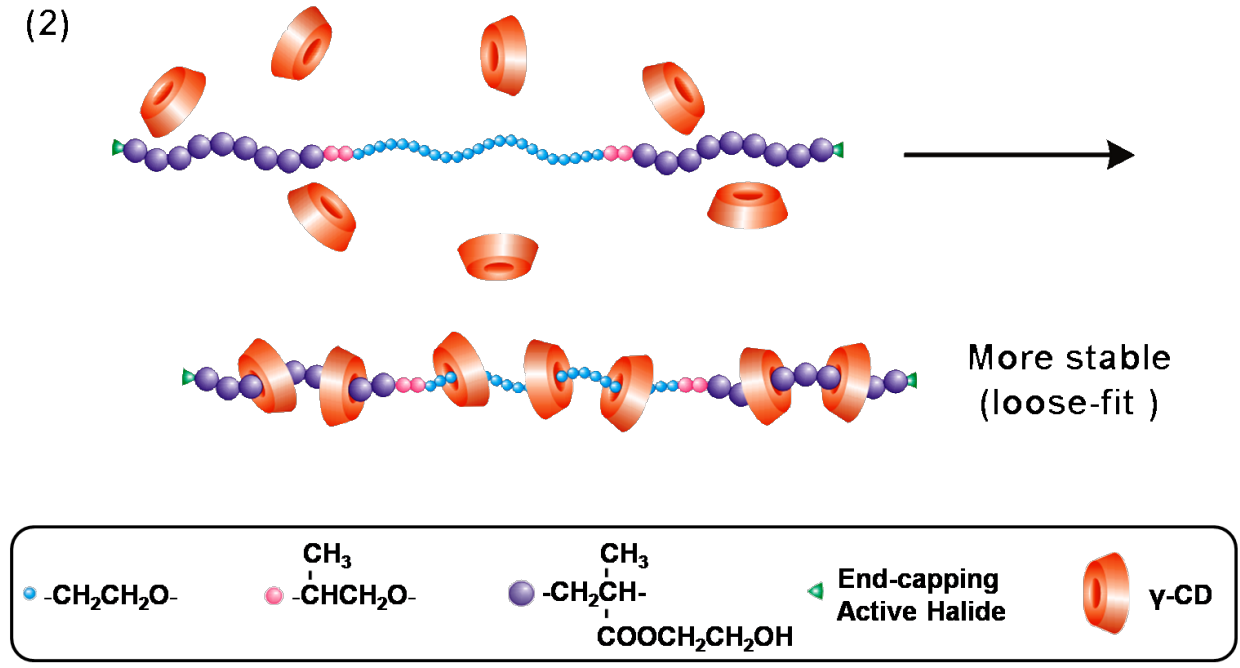


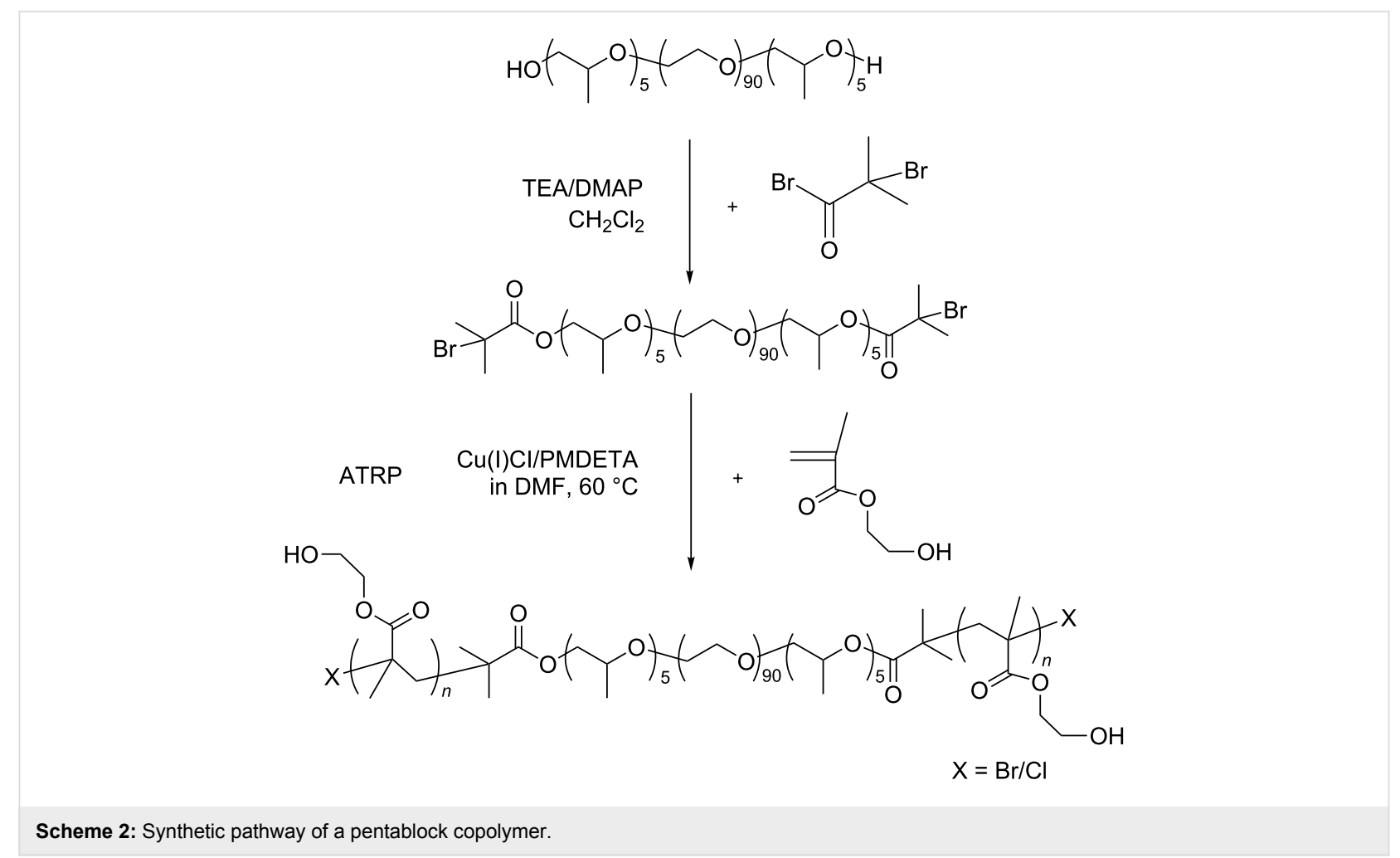

PPO, triblock copolymer was studied. The copolymer had an average degree of polymerization (DP) of five PPO flanking blocks instead of pure PEG in order to enlarge the volume of the end-capping, 2-bromoisobutyryl group. Furthermore, there is a hydrolytic side reaction of end-capped bromine in the in situ aqueous ATRP of NIPAAm that can reduce the chain end functionality and the efficiency of future chain end modification. Thus, $\mathrm{Cu}(\mathrm{I}) \mathrm{Cl} / \mathrm{PMDETA}$ was chosen as catalyst and DMF as solvent for the ATRP of HEMA in this study [19].

Table 1 summarizes the ${ }^{1} \mathrm{H}$ NMR and GPC analytical results of the pentablock copolymer. The high conversation ratio $(87 \%)$ of the monomer and the low $M_{\mathrm{w}} / M_{\mathrm{n}}$ value (1.23) in the preparation of PHEMA-PPO-PEO-PPO-PHEMA suggest that the ATRP carried out using $\mathrm{Cu}(\mathrm{I}) \mathrm{Cl} / \mathrm{PMDETA}$ as a catalyst and DMF as a solvent was successful (Supporting Information, Figure S1 and Figure S2). For convenience, the resulting macroinitiator was designated as BrPEPBr and the pentablock copolymer as PEP26M.
As illustrated in Scheme 1, both double-chain stranded, tight-fit and single-chain stranded, loose-fit PPRs are constructed from the self-assembly of $\gamma$-CDs with the macroinitiator and the pentablock copolymer in aqueous solution at room temperature. The PPRs obtained from the inclusion complexation of $\gamma$-CDs with PHEMA-PPO-PEO-PPO-PHEMA are assigned as $\mathrm{PEP} 26 \mathrm{MnCD}$, where $\mathrm{n}$ represents the feed molar ratio of $\gamma-\mathrm{CD}$ to PEP26M. Meanwhile, BrPEPBr was also self-assembled with $\gamma$-CDs (feed molar ratio of $\mathrm{BrPEPBr} / \gamma-\mathrm{CD}=1: 18$ ) in aqueous solution to provide a reference PPR labelled as PEP18CD. The theoretical and resulting compositions and yields of PEP26MnCDs and PEP18CD are summarized in Table 2. The evolution of the self-assembly of $\gamma$-CDs with the macroinitiator and pentablock copolymer is depicted in Figure 1. As can be seen, the turbidity of the PEP18CD solution is abruptly increased within several minutes. This is in contrast to several hours for PEP26MnCDs, which is consistent with the conformational differences in the double-chain and single-chain stranded PPRs. This implies that PEG tends to be bent in order

\begin{tabular}{|c|c|c|c|c|c|c|}
\hline & \multirow[t]{2}{*}{ Reaction time/h } & \multicolumn{2}{|c|}{ Molar ratio of BrPEPBr:HEMA } & \multirow[t]{2}{*}{$M_{\mathrm{n}}^{\mathrm{a}}$} & \multirow[t]{2}{*}{$M_{\mathrm{n}}^{\mathrm{b}}$} & \multirow[t]{2}{*}{$M_{\mathrm{w}} / M_{\mathrm{n}}^{\mathrm{b}}$} \\
\hline & & Feed ratio & Found ratio ${ }^{a}$ & & & \\
\hline PEP26M & 24 & $1: 30$ & $1: 26.0$ & 8260 & $9.0 \times 10^{3}$ & 1.23 \\
\hline
\end{tabular}

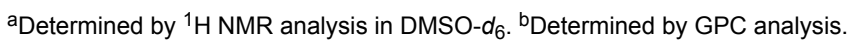


Table 2: Theoretical and resulting compositions and yields of PPRs.

\begin{tabular}{|c|c|c|c|c|}
\hline & \multirow[t]{2}{*}{ Linear guest molecule } & \multicolumn{2}{|c|}{ Molar ratio of guest molecule:y-CD } & \multirow[t]{2}{*}{ Yield $^{b}$} \\
\hline & & Feed ratio & Resulting ratio ${ }^{a}$ & \\
\hline PEP26M9CD & PEP26M & $1: 9$ & $1: 10.7$ & $22.5 \%$ \\
\hline PEP26M18CD & & $1: 18$ & $1: 16.0$ & $43.0 \%$ \\
\hline PEP26M27CD & & $1: 27$ & $1: 21.9$ & $45.1 \%$ \\
\hline PEP18CD & $\mathrm{BrPEPBr}$ & $1: 18$ & 21.3 & $72.9 \%$ \\
\hline
\end{tabular}

aDetermined by ${ }^{1} \mathrm{H}$ NMR analysis in DMSO- $d_{6}$. bYield is calculated based on the weight of precipitated PPR divided by that of all the feed materials.

to quickly pass through the inner cavity of $\gamma$-CDs when the 2-bromoisobutyryl initiating groups are attached to two ends. However, if these end-capping groups were replaced by the bulkier polymer blocks (e.g., PHEMA), the self-assembly of $\gamma$-CDs would become a time-consuming process. This is because the $\gamma$-CDs would need to accommodate and slip off the bulkier PHEMA blocks in order to distribute along the whole polymer chain, leading to the more stable, single-chain stranded, loose-fit PPRs.
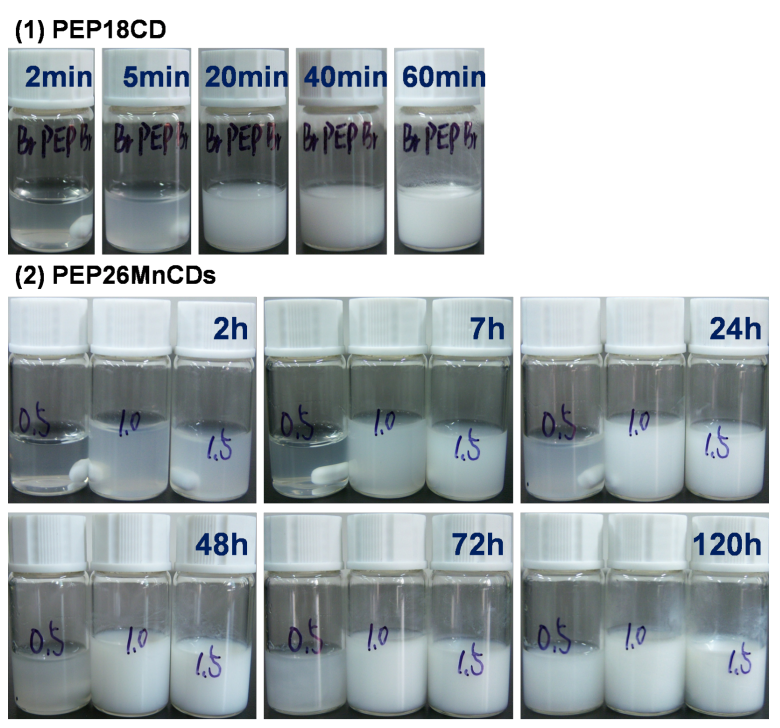

Figure 1: Photographs of the turbidity evolution in PEP18CD (1) and PEP26MnCDs (2) (PEP26M9CD (left), PEP26H18CD (middle) and PEP26M27CD (right)) at room temperature.

Furthermore, as seen in Figure 1, the turbidity of the PEP26MnCD solutions increases rapidly with the increase in the feed molar ratio of $\gamma$-CDs to PEP26M. This suggests that more $\gamma$-CDs entrap the PEP26M main chain and a faster accommodation process is accomplished in aqueous solution. The resulting molar ratio of $\gamma$-CDs to PEP26M increased from 10.7 to 21.9 when the feed molar ratio was increased from 9 to 27 , respectively. Although all of the yields in the range of $22.5 \%$ to
$45.1 \%$ appear low in this study, they are markedly higher than ever reported for single-chain stranded, loose-fit, CD-based PPRs or PRs [5,14-16]. The significant difference in yield between PEP26M18CD and PEP18CD reveals another demonstration of the reliable diversity in the loose-fit and tight-fit PPRs.

\section{Characterization of PPRs self-assembled from $\mathrm{y}$-CDs with a macroinitiator and pentablock copolymer}

The WXRD patterns of $\gamma$-CD, PEP26M, PHEMA and PPRs are presented in Figure 2. The major diffraction peaks of $\gamma$-CD appear at $2 \theta=5.1^{\circ}, 10.2^{\circ}, 12.3^{\circ}, 15.4^{\circ}, 16.4^{\circ}, 18.8^{\circ}$ and $21.7^{\circ}$, respectively, which correspond to a cage-type crystal structure [20]. The pentablock polymer PEP26M shows two strong peaks at $19.2^{\circ}$ and $23.3^{\circ}$, originating from the crystal structure of the PEO central block [21]. The pure PHEMA displays two broadfeatured peaks at $2 \theta=18.3^{\circ}, 29.2^{\circ}$ that are characteristic of an amorphous polymer. As portrayed in Scheme 1, BrPEPBr is apt to form a stable, single PEO-bent conformation in order to selfassemble with $\gamma$-CDs in aqueous solution. This shows a characteristic, channel-type, crystal structure diffraction peak at $2 \theta=7.5^{\circ}$ in accordance with our previous reports [22]. Interestingly, all of the samples resulting from the self-assembly of $\gamma$-CDs with PEP26M reveal three new broad peaks at $2 \theta=12.4^{\circ}, 17.3^{\circ}$ and $21.5^{\circ}$, but no diffraction peak at $7.5^{\circ}$. This unique diffraction pattern is quite similar to that of the singlechain stranded, loose-fit $\gamma$-CD-based PPRs or PRs as previously reported [5,14-16,23].

Due to a relatively bulkier yet appropriate cross-sectional area, the attachment of PHEMA evidently changes the self-assembly direction of $\gamma$-CDs with the pentablock copolymer as compared with the macroinitiator. In the case of self-assembly with the latter, the two parallel macroinitiator chains are not permitted to pass through the cavity of $\gamma$-CDs, and alternatively, a single PEG chain is bent into a double-chain strand. This gives rise to a tight-fit PPR exhibiting a characteristic channel-type crystal structure. However, for the self-assembly with the pentablock copolymer, an appropriately-sized chain of HEMA repeating 


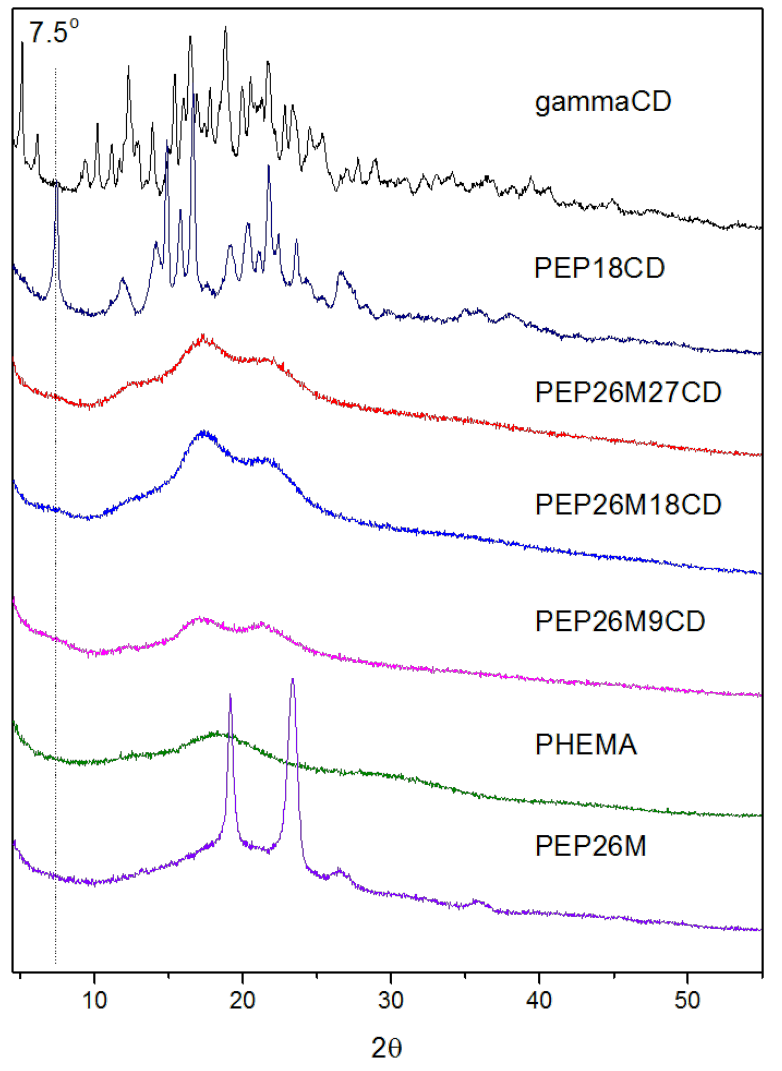

Figure 2: WXRD spectra of $\mathrm{Y}-\mathrm{CD}, \mathrm{PHEMA}, \mathrm{PEP} 26 \mathrm{M}, \mathrm{PEP} 18 \mathrm{CD}$ and PEP26MnCDs.

units enables PHEMA to be included into and to penetrate through $\gamma$-CDs. This yields more stable, single-chain stranded, loose-fit PPRs showing no characteristic channel-type crystal structure.

This single-chain stranded, loose-fit structure is also supported by ${ }^{1} \mathrm{H}$ NMR analysis. According to previous reports [12,24], the inside cavity of each $\gamma$-CD molecule could accommodate two PO or 2.2 vinyl repeating units into the resulting single-chain stranded PPRs. As outlined in Table 2, both the PHEMA and PPO blocks in PEP26M would theoretically be covered by about $16.8(10 / 2+26 / 2.2) \gamma$-CD molecules, which is less than the resulting molar ratio of 21.9 for PEP26M27CD. This is most likely caused by $\gamma$-CDs slipping into the middle PEO block. Additionally, as seen in Figure 3C, the hydroxy group resonance peaks $(\mathrm{O}(2) \mathrm{H}, \mathrm{O}(3) \mathrm{H}$ and $\mathrm{O}(6) \mathrm{H})$ of $\gamma-\mathrm{CD}$ in PEP26M27CD are clearly broader as compared with PEP26M9CD and PEP26M18CD. This is due to the decrease in conformational flexibility upon PPR formation. Furthermore, this indicates that a number of $\gamma$-CDs can still be held on the pentablock copolymer axle at a higher feed molar ratio, even in a highly polar DMSO solvent as previously described [25].
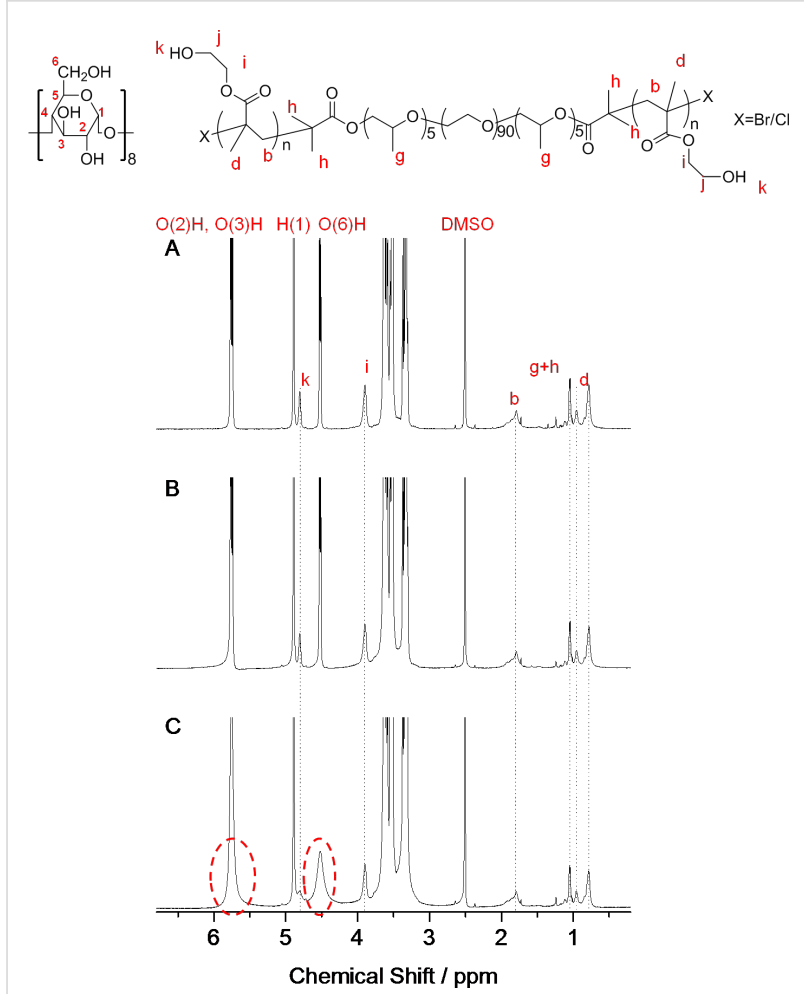

Figure 3: ${ }^{1} \mathrm{H}$ NMR spectra of PEP26M9CD (A), PEP26M18CD (B) and PEP26M27CD (C).

DSC measurements provide further evidence of the unique, single-chain stranded, loose-fit structure formed by the selfassembly of $\gamma$-CDs with PHEMA-PPO-PEO-PPO-PHEMA, as seen in Figure 4. These results clearly show endothermic peaks in PEP26M and BrPEPBr (in addition to a glass transition region in PHEMA), which correspond to the melting temperature $\left(T_{\mathrm{m}}\right)$ of the crystallized PEO segment and the glass transition temperature $\left(T_{\mathrm{g}}\right)$ of pure PHEMA, respectively. On the contrary, the curves of the PEP26MnCDs samples exhibit no obvious signal mutation from 20 to $80{ }^{\circ} \mathrm{C}$. This indicates that either both PEO and PHEMA blocks stay amorphous or that the thermal motion of chain segments in PEO and PHEMA blocks are roughly restricted due to inclusion into the cavity of $\gamma$-CDs. Owing to the formation of a single PEO-bent conformation with $\gamma$-CDs, the corresponding endothermic peak of PEO is also absent, as evidenced in PEP18CD.

TGA analysis also demonstrates the single-chain stranded, loose-fit structure of PEP26MnCDs, as depicted in Figure 5. Attributed to the protection of the $\gamma$-CD cover, the initial temperature of thermal weight loss for PEP26M27CD occurs at $260{ }^{\circ} \mathrm{C}$, which is much higher than $225^{\circ} \mathrm{C}$ for uncovered PEP26M. After the thorough thermal decomposition of covering $\gamma$-CD molecules, namely above $360{ }^{\circ} \mathrm{C}$, PEP26M27CD undergoes another distinct thermal weight loss. 


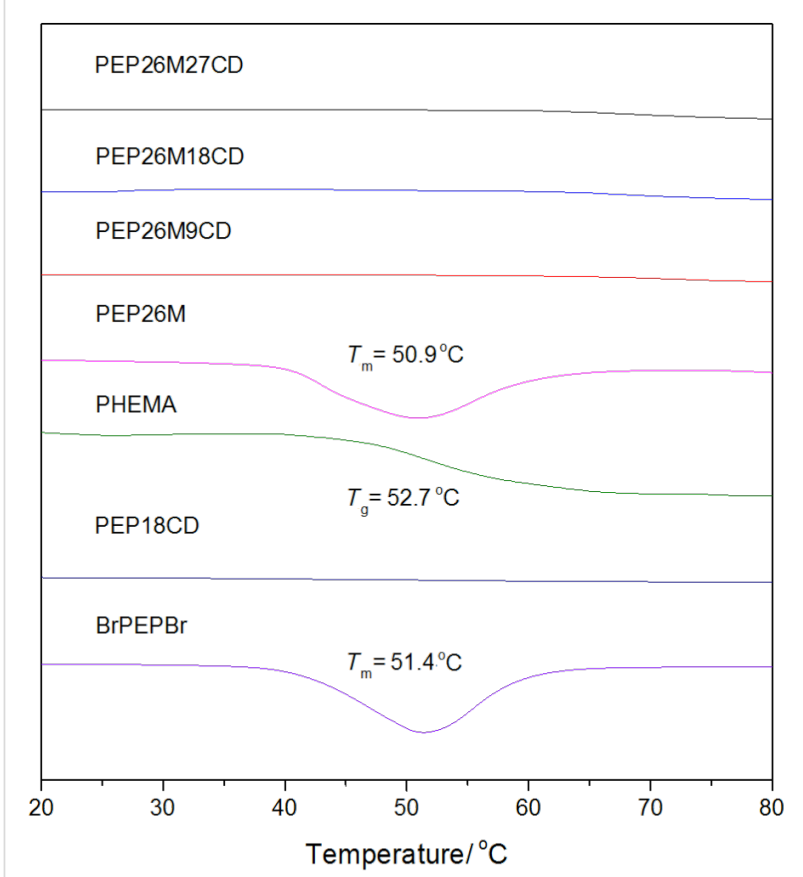

Figure 4: DSC curves of PHEMA, PEP26M, PEP26MnCDs, BrPEPBr and PEP18CD.

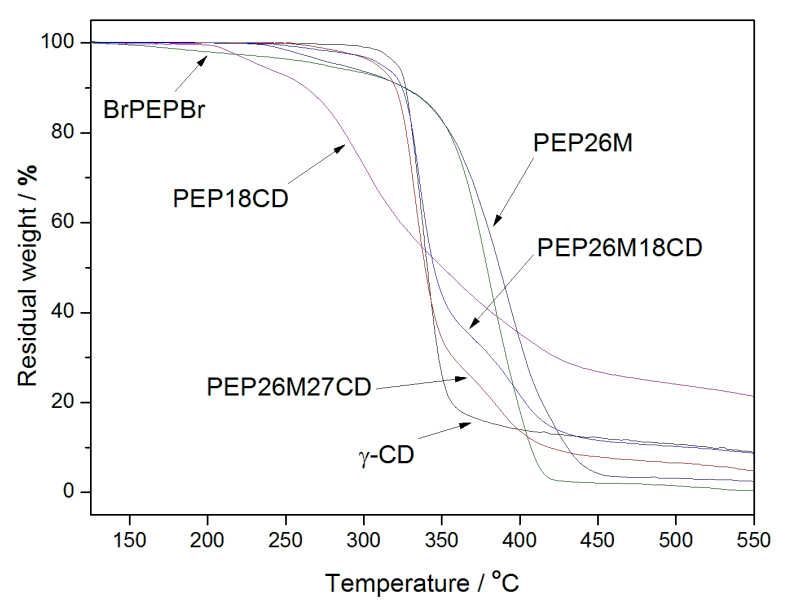

Figure 5: TGA curves of $\mathrm{Y}-\mathrm{CD}, \mathrm{PEP} 26 \mathrm{M}, \mathrm{PEP} 26 \mathrm{MnCD}$, $\mathrm{BrPEPBr}$ and PEP18CD.

This might be assigned to the decomposition of residual pentablock copolymer. Conversely, as compared with the pure $\gamma$-CD, PEP26M27CD starts to decompose at a relatively lower temperature and at a slower rate. This is similar to the behavior of the loose-fit PPR structure in which the entrapped $\gamma$-CDs are stacked in a less-ordered non-crystalline structure. The TGA curve of PEP26M18CD exhibits a similar trend. Additionally, in addition to the same shift in the initial temperature of thermal weight loss (arising from stranded $\gamma$-CDs), PEP18CD displays a unique, higher, residual weight ratio $(>20 \%)$ at $550{ }^{\circ} \mathrm{C}$, which might be credited to its tight-fit supramolecular structure.

FTIR spectroscopy analysis is a powerful technique to highlight the supramolecular structure of host and guest molecules in PPRs [26]. The FTIR spectra of PEP26MnCDs and their precursors are given in Figure 6. The spectrum of PEP26M exhibits distinct vibrational peaks around $750 \mathrm{~cm}^{-1}$ (out of plane bending of $\mathrm{C}-\mathrm{O}$ in the ester of HEMA repeat units) [27] and $1280 \mathrm{~cm}^{-1}\left(\mathrm{CH}_{2}\right.$ twist in EO repeat units) [28]. Importantly, both peaks vanish in the spectra of PEP26MnCDs, which is characteristic of the restricting and shielding effects from the inner cavity of $\gamma$-CDs to the vibrations of correlative chemical bonds. This offers supplementary (if not direct) proof of the alleged single-chain stranded, loose-fit structure of PEP26MnCDs. Similarly, PEP18CD also confirms the expected disappearance of the $\mathrm{CH}_{2}$ twist vibrational peak, which occurs when $\gamma$-CDs compactly locate on the PEO chain in a head-head or tail-tail manner.

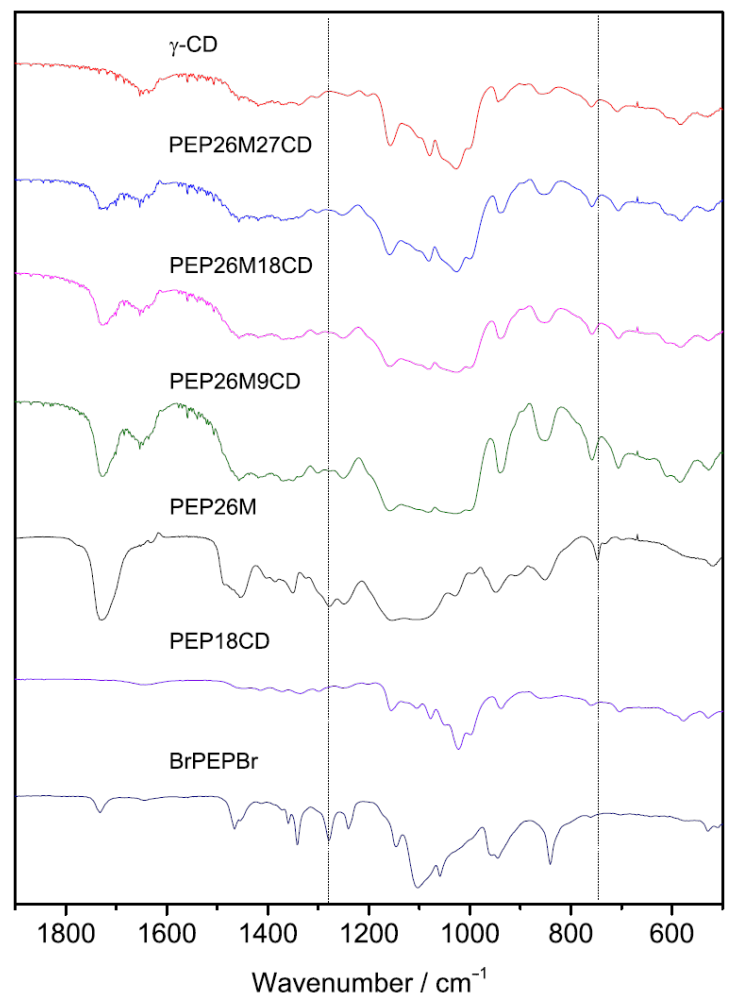

Figure 6: FTIR spectra of $\mathrm{Y}-\mathrm{CD}, \mathrm{PEP} 26 \mathrm{M}, \mathrm{PEP} 18 \mathrm{CD}$ and PEP26MnCDs.

Figure 7 compares the ${ }^{13} \mathrm{C} \mathrm{CP/MAS} \mathrm{NMR} \mathrm{spectrum} \mathrm{of}$ PEP26M27CD with those of PEP26M and $\gamma$-CD. Consistent with previous research $[29,30]$, uncomplexed $\gamma$-CDs assert less symmetrical cyclic conformations with clear multiple $\mathrm{C}_{1}, \mathrm{C}_{4}$ and $\mathrm{C}_{6}$ resonance peaks. This is in contrast to single $\mathrm{C}_{1}, \mathrm{C}_{4}$ and 
$\mathrm{C}_{6}$ resonances in PEP26M27CD, which prove that more symmetrical, cyclic $\gamma$-CDs cover and reside along the PEP26M main chain of PEP26M27CD. Noteworthy is the fact that the $-\mathrm{CH}_{3}$ resonance of PEP26M27CD similarly displays weak peak splits. This implies that the PHEMA and PPO blocks probably adopt unusual substructure conformations in the cavity of $\gamma$-CDs, different from the general morphology in the original PEP26M. As a result, they create the single-chain stranded, loose-fit structured PPRs. A further investigation focusing on molecular recognition between $\gamma$-CD and PHEMA is ongoing in our laboratory.

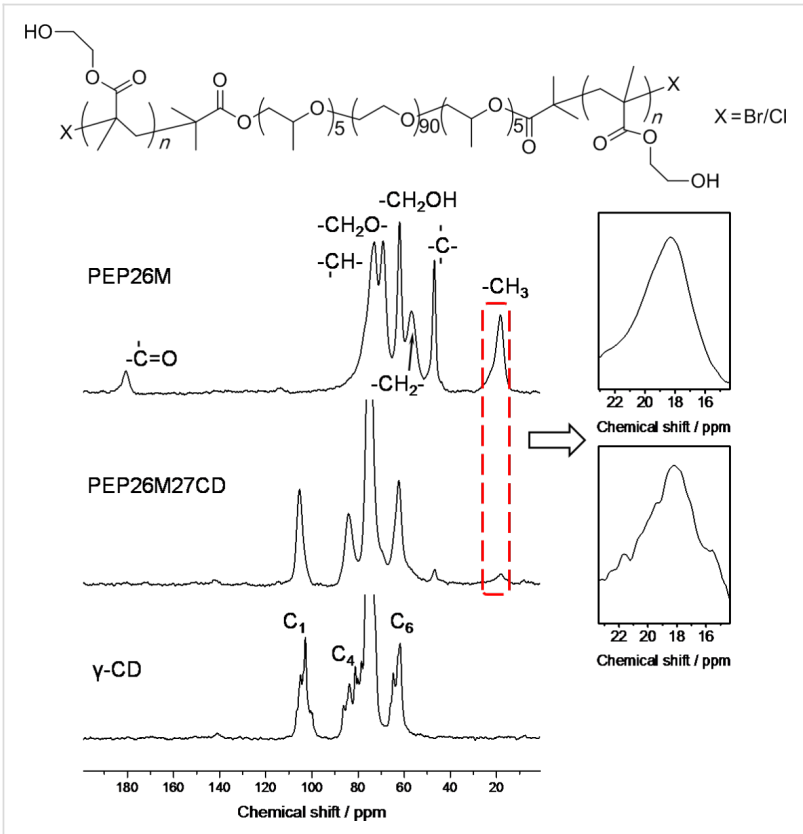

Figure 7: ${ }^{13} \mathrm{C} C P / M A S$ NMR spectra of PEP26M, $y-C D$ and PEP26M27CD.

\section{Conclusion}

A series of $\gamma$-CD-based PPRs were prepared via the selfassembly of $\gamma$-CDs with a pentablock copolymer PHEMA-PPOPEO-PPO-PHEMA in aqueous solution at room temperature. The resulting PPRs possess a unique single-chain stranded, loose-fit conformation showing no characteristic channel-type crystal structure. This finding highlights a novel model to fulfil molecular recognition between transformable $\gamma$-CDs and functional, bulkier vinyl polymers. This highlights the potential for smart material and biomedical applications. Further investigations are underway in our laboratory.

\section{Experimental}

\section{General measures}

${ }^{1} \mathrm{H}$ NMR (400 MHz) spectra were recorded on a Bruker ARX400 spectrometer at room temperature using DMSO- $d_{6}$ as a solvent and tetramethylsilane (TMS) as an internal standard.
${ }^{13} \mathrm{C}$ cross-polarization magic-angle spinning (CP/MAS) NMR spectra were measured on a Bruker AV 300 NMR spectrometer with a single contact time of $1 \mathrm{~ms}$ and a spinning rate of $5 \mathrm{kHz}$. Chemical shifts were compared to an external adamantane standard. Gel permeation chromatographic (GPC) measurements were carried out at $40{ }^{\circ} \mathrm{C}$ on a HLC-8320GPC (TOSOH, Japan) instrument using THF as eluent at a flow rate of $0.3 \mathrm{~mL} / \mathrm{min}$. All of the GPC data were calibrated using polystyrene (PS) standards. Fourier transform infrared spectroscopy (FTIR) spectra were measured using a Shimadzu IR Prestige-21 FTIR spectrometer at room temperature in the range between 4000 and $500 \mathrm{~cm}^{-1}$, with a resolution of $2 \mathrm{~cm}^{-1}$ and 25 averaged scans. Samples were prepared by mixing with dry $\mathrm{KBr}$ powder. Differential scanning calorimetry (DSC) measurements were run on a SHIMADZU DSC-60 differential scanning calorimeter with a scan temperature range from 20 to $80^{\circ} \mathrm{C}$ at a scan rate of $10{ }^{\circ} \mathrm{C} \min ^{-1}$ and purged with nitrogen. The samples were encapsulated in hermetically-sealed aluminum pans, and underwent two, 20 to $80{ }^{\circ} \mathrm{C}$ heating procedures. Data were collected during the second heating run. TGA was performed with a TA SDT 2960 instrument at a heating rate of $10{ }^{\circ} \mathrm{C} \mathrm{min}-1$ while purged with nitrogen, and the temperature was scanned from ambient temperature to $550{ }^{\circ} \mathrm{C}$. Wide-angle X-ray diffraction (WXRD) measurements were carried out with powder samples using a Shimadzu XD-D1 X-ray diffractometer. The radiation source was Ni-filtered, $\mathrm{Cu} \mathrm{K} \alpha$ radiation with a wavelength of $0.154 \mathrm{~nm}$. The voltage was set to $40 \mathrm{kV}$ and the current to $40 \mathrm{~mA}$. Samples were placed on a sample holder and scanned from $2 \theta=4.5$ to $60^{\circ}$ at a speed of $5^{\circ} \mathrm{min}^{-1}$.

\section{Materials}

$\gamma$-CD (Wako, Japan) and PPO-PEO-PPO (comprised of a central block of 90 PEO units and two flank blocks of 5 PO units having $M_{\mathrm{n}}=4580$ (Zhejiang Huangma Chemical Industry Group Co., Ltd, China)) were used as received without further purification. 2-Hydroxyethyl methacrylate (HEMA) (TCI, Japan) was passed over a short basic alumina column to remove the inhibitor before polymerization. $N, N, N$ ' $N$ ', $N$ '-pentamethyldiethylenetriamine (PMDETA) and ethyl 2-bromoisobutyrate were purchased from Sigma, USA. Both 2-bromoisobutyryl bromide and 4-dimethylaminopyridine (DMAP) were available from Alfa Aesar, USA. Triethylamine (TEA) (VAS Chemical Reagents Company, China) was refluxed with $p$-toluenesulfonyl chloride and distilled under vacuum. Copper(I) chloride $(\mathrm{Cu}(\mathrm{I}) \mathrm{Cl})$ was prepared from $\mathrm{CuCl}_{2}$, purified by stirring in hydrochloric acid, washed with methanol and finally dried under vacuum prior to use. $\mathrm{CH}_{2} \mathrm{Cl}_{2}$ was stirred with $\mathrm{CaH}_{2}$ and distilled under reduced pressure. DMF was supplied by Sinopharm Chemical Reagent Company, China and used without further purification. All other solvents and reagents were of analytical grade. 


\section{Synthesis of 2-bromoisobutyryl end-capped PPO-PEO-PPO (BrPEPBr)}

PPO-PEO-PPO was converted to the corresponding ATRP macroinitiator through the end-capping reaction with a fourfold molar excess of 2-bromoisobutyryl bromide in $\mathrm{CH}_{2} \mathrm{Cl}_{2}$. PPOPEO-PPO (9.16 g, $2 \mathrm{mmol}$ ), DMAP (488 $\mathrm{mg}, 4 \mathrm{mmol}$ ) and TEA (404 mg, $4 \mathrm{mmol}$ ) were dissolved in $20 \mathrm{~mL} \mathrm{CH}_{2} \mathrm{Cl}_{2}$ in a $100 \mathrm{~mL}$ three-neck round-bottom flask. Thereafter, another $20 \mathrm{~mL}$ of $\mathrm{CH}_{2} \mathrm{Cl}_{2}$ containing 2-bromoisobutyryl bromide $(1.00 \mathrm{~mL}, 8 \mathrm{mmol})$ was added drop-wise under nitrogen. The reaction continued for $2 \mathrm{~h}$ at $0{ }^{\circ} \mathrm{C}$ and then for another $24 \mathrm{~h}$ at room temperature under stirring. Finally, the mixture was filtered to remove the precipitated bromide salt. The product was purified by precipitation into $500 \mathrm{~mL}$ of anhydrous ether at $10{ }^{\circ} \mathrm{C}$. The sequence was repeated three times. ${ }^{1} \mathrm{H}$ NMR analysis indicated that the degree of esterification was $>99 \%$, and the yield was $83.4 \%$ (Figure S3, Supporting Information File 1).

\section{Synthesis of PHEMA-PPO-PEO-PPO- PHEMA via ATRP}

A typical procedure for the synthesis of the PHEMA-PPO-PEOPPO-PHEMA pentablock copolymer via ATRP of HEMA was as follows. In a sealable Pyrex reactor, $\operatorname{BrPEPBr}(0.488 \mathrm{~g}$, $0.1 \mathrm{mmol})$ was dissolved in $4 \mathrm{~mL}$ DMF. HEMA (0.39 $\mathrm{g}$, $3.0 \mathrm{mmol}$ ) dissolved in $2 \mathrm{~mL}$ of DMF was added to this mixture, and then PMDETA $(69.3 \mathrm{mg}, 0.4 \mathrm{mmol})$ was added. The mixture was degassed by three freeze-pump-thaw cycles, then quenched in liquid nitrogen to which $\mathrm{Cu}(\mathrm{I}) \mathrm{Cl}(39.6 \mathrm{mg}$, $0.4 \mathrm{mmol}$ ) was added. The reactants in the reactor were degassed three times by purging with nitrogen. The reactor was sealed under vacuum and the reaction was maintained for $24 \mathrm{~h}$ at $60{ }^{\circ} \mathrm{C}$ under stirring. The polymerization was stopped after breaking the Pyrex reactor, and the product was dialyzed using a cellulose membrane (molecular weight cut-off $(\mathrm{MWCO})=$ 3500 ) and lyophilized, resulting in a yield of $84.3 \%$.

\section{Synthesis of PHEMA via ATRP}

As previously reported [31], PHEMA (DP $=29$, PDI $=1.19)$ was synthesized by ATRP of HEMA in a DMF/ $\mathrm{H}_{2} \mathrm{O}$ $(\mathrm{w} / \mathrm{w}=1: 1)$ mixture at $25^{\circ} \mathrm{C}$ using ethyl 2-bromoisobutyrate as an initiator, $\mathrm{Cu}(\mathrm{I}) \mathrm{Cl}$ as a catalyst and PMDETA as a ligand.

\section{Preparation of PPRs from the self-assembly of $\mathrm{Y}$-CDs with a pentablock copolymer}

A protocol for the preparation of PPRs built from the selfassembly of PHEMA-PPO-PEO-PPO-PHEMA with a varying amount of $\gamma$-CDs was as follows. A saturated aqueous solution containing a predetermined amount of $\gamma$-CDs was added to a $1.5 \mathrm{~mL}$ aqueous solution of the pentablock copolymer $(0.10 \mathrm{~g}$, $\left.1.21 \times 10^{-2} \mathrm{mmol}\right)$, followed by vigorous stirring at room temperature for sufficient time to form a PPR. The resulting PPR was collected by centrifugation and washed with a small amount of water to remove residual free $\gamma$-CDs before freezedrying.

\section{Supporting Information}

Supporting Information File 1 contains a GPC trace of PEP26M and ${ }^{1} \mathrm{H}$ NMR spectra of PEP26M and BrPEPBr.

\section{Supporting Information File 1}

Additional GPC trace and ${ }^{1} \mathrm{H}$ NMR spectral data.

[http://www.beilstein-journals.org/bjoc/content/ supplementary/1860-5397-10-257-S1.pdf]

\section{Acknowledgements}

The authors acknowledge support from the Natural Science Foundation of China (No. 20974015) and the Doctoral Program Foundation of the Ministry of Education of

China (No. 20091101110029).

\section{References}

1. Harada, A.; Hashidzume, A.; Takashima, Y. Adv. Polym. Sci. 2006, 201, 1-43. doi:10.1007/12_056

2. Kida, T.; Kikuzawa, A.; Akashi, M. Chem. Lett. 2008, 37, 1120-1121. doi:10.1246/cl.2008.1120

3. Jing, Z.-y.; Xu, X.-m.; Chen, H.-q.; Li, X.-h. Cyclodetrin chemistry: preparation and application; Chemical Industry Press: Beijing, 2009; pp $7 \mathrm{ff}$.

4. Harada, A.; Li, J.; Kamachi, M. Nature 1994, 370, 126-128. doi: $10.1038 / 370126 a 0$

5. Takahashi, A.; Katoono, R.; Yui, N. Macromolecules 2009, 42, 8587-8589. doi:10.1021/ma9022372

6. Harada, A.; Hashidzume, A.; Yamaguchi, H.; Takashima, Y. Chem. Rev. 2009, 109, 5974-6023. doi:10.1021/cr9000622

7. San-Miguel, V.; González, M.; Pozuelo, J.; Baselga, J. Polymer 2009, 50, 5884-5891. doi:10.1016/j.polymer.2009.10.052

8. Li, S.; Wang, J.; Jiang, L.; Ye, L.; Zhang, A.; Feng, Z. Chin. J. Chem. 2012, 30, 2453-2460. doi:10.1002/cjoc.201200280

9. Ren, L.; Ke, F.; Chen, Y.; Liang, D.; Huang, J. Macromolecules 2008, 41, 5295-5300. doi:10.1021/ma800632m

10. Huang, F.; Gibson, H. W. Prog. Polym. Sci. 2005, 30, 982-1018. doi:10.1016/j.progpolymsci.2005.07.003

11. Zhang, Z.-X.; Liu, K. L.; Li, J. Macromolecules 2011, 44, 1182-1193. doi:10.1021/ma102196q

12. Fan, M. M.; Zhang, X.; Qin, J.; Li, B.-J.; Sun, X.; Zhang, S. Macromol. Rapid Commun. 2011, 32, 1533-1538. doi:10.1002/marc.201100272

13. Tong, X.-m.; Hou, D.-d.; Zhang, X.-w.; Ye, L.; Zhang, A.-y.; Feng, Z.-g. Chin. J. Polym. Sci. 2008, 26, 723-732. doi:10.1142/S0256767908003473

14. Jiang, L.; Ye, L.; Zhang, A.-y.; Feng, Z.-g. Macromol. Chem. Phys. 2014, 215, 1022-1029. doi:10.1002/macp.201400047 
15. Wang, J.; Li, S.; Ye, L.; Zhang, A.-Y.; Feng, Z.-G. Macromol. Rapid Commun. 2012, 33, 1143-1148. doi:10.1002/marc.201200017

16. Gao, P.; Wang, J.; Wang, P.-J.; Ye, L.; Zhang, A.-Y.; Feng, Z.-G. Macromol. Chem. Phys. 2012, 212, 1532-1539. doi:10.1002/macp.201200105

17. Tong, X.; Zhang, X.; Ye, L.; Zhang, A.-y.; Feng, Z.-g. Polymer 2008, 49, 4489-4493. doi:10.1016/j.polymer.2008.08.036

18. Zhou, Y.; Fan, X.; Xue, D.; Xing, J.; Kong, J. React. Funct. Polym. 2013, 73, 508-517. doi:10.1016/j.reactfunctpolym.2012.12.001

19. Maier, S.; Sunder, A.; Frey, H.; Mülhaupt, R. Macromol. Rapid Commun. 2000, 21, 226-230. doi:10.1002/(SICI)1521-3927(20000301)21:5<226::AID-MARC226>3.0 .CO;2-O

20. Wang, J.; Ye, L.; Zhang, A.-y.; Feng, Z.-g. J. Mater. Chem. 2011, 21, 3243-3250. doi:10.1039/c0jm02803g

21. Wang, J.; Gao, P.; Ye, L.; Zhang, A.-y.; Feng, Z.-G. J. Phys. Chem. B 2010, 114, 5342-5349. doi:10.1021/jp101068b

22. Gao, P.; Wang, J.; Ye, L.; Zhang, A.-y.; Feng, Z.-g. Macromol. Chem. Phys. 2011, 212, 2319-2327. doi:10.1002/macp.201100319

23. Li, J.; Yan, D.; Chen, Q. Sci. China, Ser. B: Chem. 2002, 45, 73-83.

24. Harada, A.; Okada, M.; Li, J.; Kamachi, M. Macromolecules 1995, 28 , 8406-8411. doi:10.1021/ma00128a060

25. Zhao, T.; Beckham, H. W. Macromolecules 2003, 36, 9859-9865. doi:10.1021/ma035513f

26. Miura, T.; Kida, T.; Akashi, M. Macromolecules 2011, 44, 3723-3729. doi:10.1021/ma200257z

27. Perova, T. S.; Vij, J. K.; Xu, H. Colloid Polym. Sci. 1997, 275, 323-332. doi:10.1007/s003960050089

28. Su, Y.-I.; Wang, J.; Liu, H.-z. Macromolecules 2002, 35, 6426-6431. doi: $10.1021 / \mathrm{ma} 0105284$

29. Huh, K. M.; Ooya, T.; Lee, W. K.; Sasaki, S.; Kwon, I. C.; Jeong, S. Y.; Yui, N. Macromolecules 2001, 34, 8657-8662. doi:10.1021/ma0106649

30. Huh, K. M.; Ooya, T.; Sasaki, S.; Yui, N. Macromolecules 2001, 34, 2402-2404. doi:10.1021/ma0018648

31. Robinson, K. L.; Khan, M. A.; de Paz Báñez, M. V.; Wang, X. S.; Armes, S. P. Macromolecules 2001, 34, 3155-3158. doi:10.1021/ma0019611

\section{License and Terms}

This is an Open Access article under the terms of the Creative Commons Attribution License (http://creativecommons.org/licenses/by/2.0), which permits unrestricted use, distribution, and reproduction in any medium, provided the original work is properly cited.

The license is subject to the Beilstein Journal of Organic Chemistry terms and conditions: (http://www.beilstein-journals.org/bjoc)

The definitive version of this article is the electronic one which can be found at: doi:10.3762/bjoc. 10.257 